\title{
Electron Energy-Loss Spectroscopy of Ceria Abrasives
}

\author{
Shelley R. Gilliss, James Bentley ${ }^{\S}$ and C. Barry Carter \\ Dept. of Chemical Engineering and Materials Science, University of Minnesota, \\ 421 Washington Ave. S.E., Minneapolis, MN 55455, USA \\ ${ }^{\S}$ Metals and Ceramics Division, Oak Ridge National Laboratory, PO Box 2008, Oak Ridge, \\ TN 37830-6064, USA
}

Cerium oxide (ceria: $\mathrm{CeO}_{2}$ ) is one of the most widely used abrasives for the chemical-mechanical polishing (CMP) of silicate glasses. The common occurrence of "impure" ceria abrasives as effective polishing compounds has led to much speculation on the role of impurities on the "C" (chemical component) in CMP. Typically, when the chemical composition of ceria abrasives is determined, the method used describes only the bulk overall composition and does not yield a spatially related understanding of the composition. The current investigation analyzes the chemistry of ceria abrasives (both before and after polishing) at the nanometer scale and elucidates the degree of chemical homogeneity in a typical abrasive. The ceria abrasives were investigated using electron energy-loss spectroscopy (EELS) in a Philips CM200FEG field-emission gun transmission electron microscope equipped with a Gatan imaging filter (GIF). Spectra were acquired and processed with Emispec Vision and also exported for further analysis with Gatan EL/P software. It is well established that EELS can be a powerful tool for identifying oxidation state, bonding and crystal structure in materials $[1,2]$.

Analysis of ceria abrasives with vastly different cut rates and physical appearance is the focus of this study. For example, a "ceria abrasive" can vary dramatically in physical appearance, ranging in color from white to a deep red. The spatial resolution of the chemical information gathered by EELS is largely determined by the size of the probe. With the typical probe of diameter $\sim 1.5 \mathrm{~nm}$ (full width at half maximum) containing $\sim 1$ nano Amp, EELS data could routinely be obtained from the individual constituent grains of the complex polycrystalline particles (Figure 1). Particles were probed in two different abrasives and both abrasives were found to be chemically heterogeneous. For example, in one sample of ceria abrasive, individual particles containing only $\mathrm{Al}$ and $\mathrm{O}$, or only $\mathrm{Si}$ and $\mathrm{O}$, besides the expected particles of only $\mathrm{Ce}$ and $\mathrm{O}$ were detected. The presence of alumina and silica is likely due to fillers added by the manufacturer. In a different sample of ceria abrasive, "pure" ceria particles were rarely seen; most contained minor levels of elements such as Ba, La, and $\mathrm{Pr}$, as shown in Figure 2. Fluorine was also sometimes detected. Figure 2 shows an example of a spectrum from a La- and F-rich particle, containing smaller amounts of $\mathrm{Ce}, \mathrm{Pr}$ and $\mathrm{O}$. Cerium-rich particles containing $\mathrm{F}$ and $\mathrm{O}$ were also detected (Figure 3). Qualitatively, the reduced oxygen signal in the spectrum from the F-containing particle is apparent. Although complicated by the presence of overlapping La, Ce and $\operatorname{Pr} \mathrm{M}_{45}$ edges, conventional quantitative analysis of the spectra in Figure 3 yielded compositions of $(\mathrm{La}+\mathrm{Ce}+\mathrm{Pr})_{34} \mathrm{O}_{66}$ and $(\mathrm{La}+\mathrm{Ce}+\mathrm{Pr})_{32} \mathrm{~F}_{28} \mathrm{O}_{40}$. Differences in fine structure between the two spectra in Figure 3 are also present. The twin local maxima at $\sim 531$ and $536 \mathrm{eV}$ present in the oxygen edge from $\mathrm{CeO}_{2}$ are replaced by a single broad maximum in the F-containing material. At the Ce $\mathrm{M}_{45}$ edge an increase in the $\mathrm{M}_{5} / \mathrm{M}_{4}$ white-line intensity (WLI) ratio and a shift of $\sim 1 \mathrm{eV}$ to a smaller binding energy was consistently observed for F-containing particles. Confidence in these shifts in edge onset was provided by the use of the carbon edge (from the support film) for energy-scale calibration. Note that in Figure 3 there is no shift in the La $\mathrm{M}_{45}$ white lines. The changes in WLI ratio and edge onsets are both consistent with the presence of Ce in a 3+ oxidation state rather than the 4+ state expected for $\mathrm{CeO}_{2}$. However, since mixed valences are almost certainly present, a rigorous analysis will require careful measurements from standard materials, acquisition of spectra at greater dispersion (and energy resolution) to measure energy shifts better, and sophisticated extraction of white line intensities. Fluorine's ability to stabilize the $3+$ state of the cerium atoms may be critical in the chemical activity of the abrasive. There is much speculation on the role of fluorine in ceria abrasives. Sabia and Stevens conducted polishing performance tests on 
ceria abrasives with and without fluorine. They determined the fluorine-containing abrasives had higher cut-rates than the non-fluorine containing abrasives [3]. EELS is proving to be a powerful tool for studying the chemical distribution of species in these real-world ceria abrasive materials at the nanoscale and is expected to provide even more useful information on bonding and oxidation state in future work [4].

\section{References}

1. J.H. Paterson and O.L. Krivanek, Ultramicroscopy 32 (1990) 319.

2. Rask, J.H., B.A. Miner, and P.R. Buseck, Ultramicroscopy 21 (1987) 321.

3. Sabia, R. and H.J. Stevens. Mach. Sci. Tech. 4(2) (2000) 235.

4. Funding for this research is supported by the National Science Foundation through grant NSFDMR-0104198. Research at the ORNL SHaRE Collaborative Research Center was supported by the Division of Materials Sciences and Engineering, U.S. DOE, under contract DE-AC0500OR22725 with UT-Battelle, LLC.

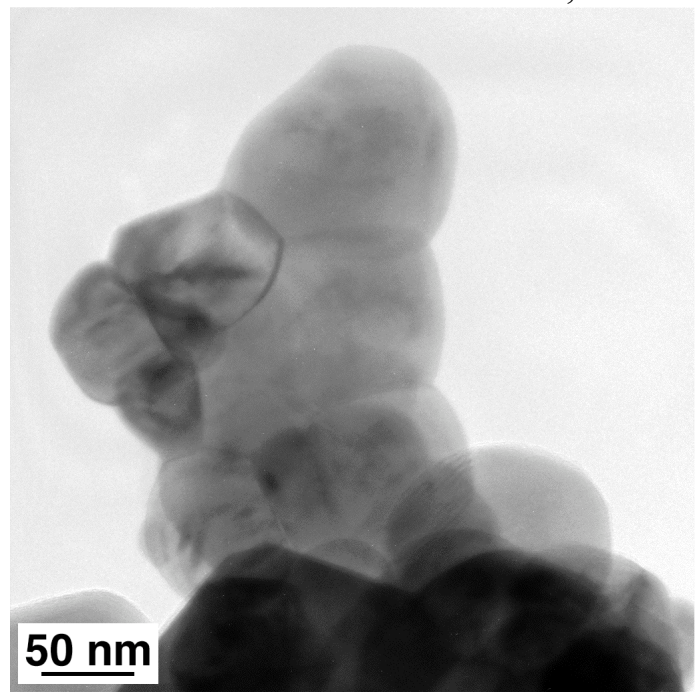

FIG. 1. TEM image showing structure typical for analyzed ceria.

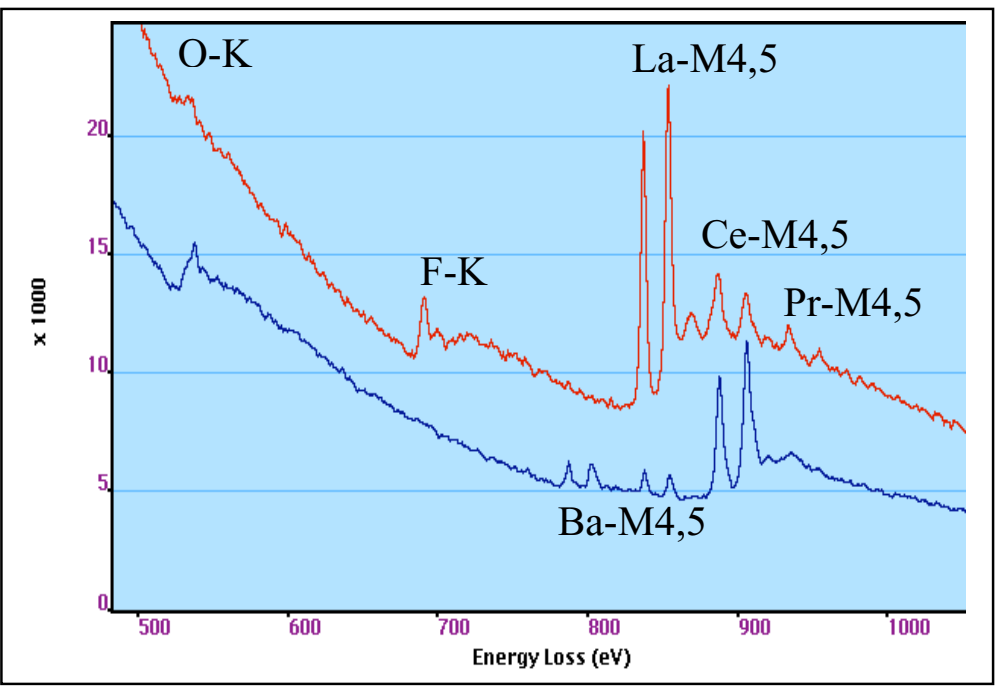

FIG. 2. EELS data from (blue) ceria particle containing La and $\mathrm{Ba}$ and (red) La- and F-rich particle with $\mathrm{Ce}, \mathrm{Pr}$ and $\mathrm{O}$

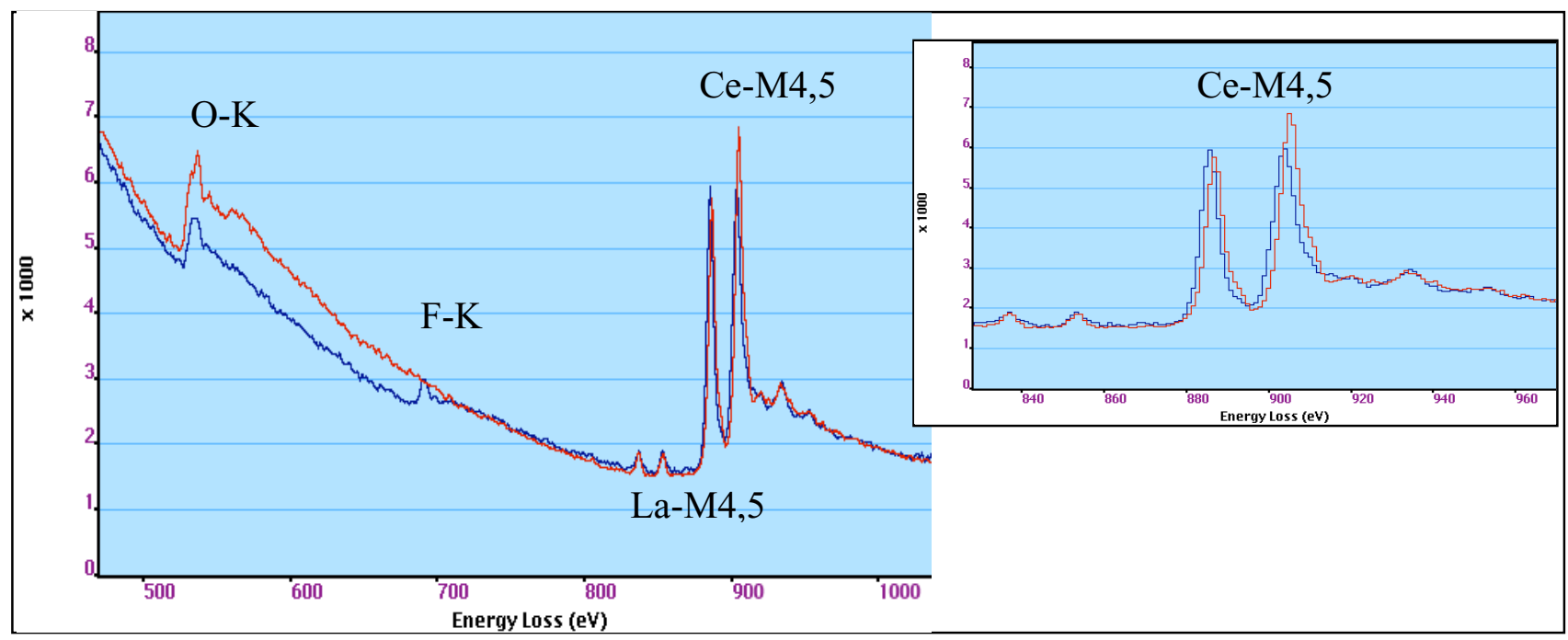

FIG. 3. EELS data from (red) $(\mathrm{La}+\mathrm{Ce}+\mathrm{Pr})_{34} \mathrm{O}_{66}$ particle and and (blue) $(\mathrm{La}+\mathrm{Ce}+\mathrm{Pr})_{32} \mathrm{~F}_{28} \mathrm{O}_{40}$ particle. Inset shows increased $\mathrm{M}_{5} / \mathrm{M}_{4}$ WLI ratio and lower edge onset energy for $\mathrm{Ce}$ in $(\mathrm{La}+\mathrm{Ce}+\mathrm{Pr})_{32} \mathrm{~F}_{28} \mathrm{O}_{40}$. 